\title{
COMUNICACIÓN
}

\section{Ectoparásitos de la perdiz chilena (Nothoprocta perdicaria) en la provincia de Nuble, Chile}

\author{
DANIEL GONZÁLEZ*, ARWID DAUGSCHIES**, KLAUS POHLMEYER***, LUIS RUBILAR*, \\ OSCAR SKEWES * y EBERHARD MEY****
}

\section{ECTOPARASITES IN CHILEAN TINAMOU (Nothoprocta perdicaria) IN ÑUBLE PROVINCE, CHILE}

\begin{abstract}
Between November 1994 and April 1995 a total of 51 chilean tinamou (Nothoprocta perdicaria) were collected in Nuble Province (Chile) and the following ectoparasites prevalences were found; Rhynchothura teres (9.2\%), Heptapsogaster sp. (90.2\%), Menacanthus nothoproctae (39.2\%), Heptapsogaster testudo (19.6\%), Allosathes sp. (80.4\%), Amblyomma sp. (11.8\%) and a mite from the Trombiculidae family (15.7\%). Excepting R. teres, the other ectoparasites represent their first record in the chilean tinamou.
\end{abstract}

Key words: Chilean tinamou, Nothoprocta perdicaria, Survey, ectoparasites, Chile.

\section{INTRODUCCIÓN}

La perdiz chilena, Nothoprocta perdicaria (Kittlitz, 1830) (Tinamiformes: Tinamidae), es un ave silvestre endémica en Chile que se distribuye desde Atacama a Llanquihue. Habita praderas semiáridas, trigales observándose frecuentemente a orillas de camino ${ }^{1}$. En estado adulto alcanza $420 \mathrm{~g}$ de peso $^{2}$. Tiene un largo corporal de $32 \mathrm{~cm}$, siendo su coloración, en general críptica ${ }^{3}$. Posee una dieta mixta, que incluye materia vegetal y pequeños animales, particularmente invertebrados ${ }^{4}$. En un estudio sobre endoparásitos de la perdiz se describió en su intestino delgado, el cestodo Aploparaksis tinamoui, ${ }^{5} \mathrm{y}$ en ejemplares colectados en invierno en Ñuble se encontraron además los helmintos
Capillaria caudinflata, Subulura suctoria y ooquistes de Eimeria sp $^{6}$. Hasta la fecha, se ha registrado como presente en esta ave sólo un ectoparásito, el Phthiraptera Rhynchothura teres $^{7}$.

\section{MATERIAL Y MÉTODO}

En la provincia de Ñuble, VIII Región de Chile, desde Noviembre de 1994 hasta Abril de 1995 se obtuvieron mediante caza autorizada por el Servicio Agrícola y Ganadero (SAG), 51 perdices ( $N$. perdicaria). Las aves en forma individual fueron depositadas inmediatamente después de cazadas en bolsas herméticas individuales.

Los ectoparásitos se aislaron en forma manual

\footnotetext{
* Departamento Ciencias Pecuarias, Facultad de Medicina Veterinaria, Universidad de Concepción, Casilla 537, Chillán, Chile.

** Instituto de Parasitología, Escuela de Med. Veterinaria, Universidad de Leipzig, Leipzig, an der Tierklinik 33 , D-04103 Leipzig, Alemania.

*** Instituto de Investigaciones Silvestres, Escuela de Med Veterinaria de Hannover, Bünteweg 17, D-30559, Hannover, Alemania.

**** Museo Nacional de Historia Natural de Turinguen, An der Brücke 3, D-07407 Rudolstadt, Alemania.
} 
desde las plumas bajo lupa estereoscópica y, luego depositados en frascos con alcohol al $70 \%$. Los Phithirapteros se limpiaron con $\mathrm{KOH}$ al 20\%, y luego sometidos a soluciones ascendentes de alcoholes $(40 \%, 80 \%$, y 100\%). Se aclararon durante 24 horas en aceite de clavo, y posteriormente fueron montados en bálsamo de Canadá ${ }^{8}$ Los ácaros fueron aclarados en solución Nesbitt por 72 horas y posteriormente montados en preparación permanente con solución Berlesse?.

\section{RESULTADOS Y DISCUSIÓN}

De las 51 aves analizadas, cincuenta $(98 \%)$ presentaban algún ectoparásito. Los parásitos de la clase Insecta fueron los hallazgos más prevalentes $(98 \%)$. Aquellos de la clase aracnida se aislaron en 44 aves $(86,3 \%)$. En general se presentaron parasitosis mixtas, las que en algunos casos fue de hasta cinco especies distintas por ave. De la ectofauna aislada, se encontraron cuatro de la clase insecta (Lamprocorpus sp., Heptapsogaster sp., Menacanthus nothoproctae y Heptapsogaster testudo) y tres especies de la clase aracnida (Allosathes sp., Amblyomma sp. y ácaros de la familia Trombiculidae).

Las prevalencias de infección expresadas en porcentaje (\%) y las intensidades de las infecciones se presentan en la Tabla 1 y el número de ejemplares colectados y su distribución según el sexo de los parásitos, el estado de desarrollo y la relación hembra-macho se presenta en la Tabla 2.

La presencia de $H$. testudo y de $R$. teresya ya había sido descrita en 1937 en Perú en el tinamiforme Nothura maculosa peruviana ${ }^{7}$, y la de $M$. nothoprocta (Phthiraptera, Menoponidae) en Nothoprocta cinerascens colectadas en Bolivia ${ }^{10}$. En el presente estudio todos ellos se encontraron con bastante frecuencia e intensidad en la perdiz chilena. (Tabla 1).

Acaros del género Allosathes (Acaridida, Crypturoptidae) se aislaron un máximo de 69 ejemplares por ave, obteniendo una media de 11,3 ácaros (Tabla 1), siendo, el parásito de mayor frecuencia en presentación. Como especie tipo de Allosathes se ha descrito a la especie $A$. anepiandrius, aislada del tinamiforme Nothura boraquira provenientes de Brasil ${ }^{11}$. Es posible que la especie encontrada en la perdiz chilena corresponda a la misma especie. De los 374 ácaros adultos aislados 250 eran hembras y 124 machos, (relación hembra-macho de 1:0,5) (Tabla. 2). Esta diferencia a favor de las hembras podrían deberse a que, en ciertos grupos de artrópodos parásitos, los machos poseen un periodo de vida más corto que la hembra, y además, en el caso de Allosathes, los machos son más pequeños y activos que la hembra, por lo que serían más difíciles de aislar ${ }^{12}$.

Larvas de los ácaros de la familia Trombiculidae fueron aislados desde el conducto auditivo externo en ocho aves $(15,7 \%)$. Se aisló un máximo de 4 especímenes en una ave, A esta familia corresponden los ácaros, cuyas larvas parásitas se denominan "ácaros estivo-otoñales", que se caracterizan porque sus ninfas y adultos son de vida libre. Se describen como parásitos de varios animales y también del hombre, y en algunos casos pueden transmitir importantes enfermedades ${ }^{13}$.

En seis aves se aislaron 51 ejemplares del genero Amblyomma, 48 ninfas y 3 larvas,

Tabla 1. Prevalencia e intensidad de ectoparásitos aislados de 51 Nothoprocta perdicaria en la provincia de $\tilde{\text { Nuble (Chile) }}$

\begin{tabular}{|c|c|c|c|c|}
\hline \multirow[b]{2}{*}{ Ectoparasito } & \multicolumn{2}{|c|}{ Prevalencia } & \multicolumn{2}{|c|}{ Intensidad } \\
\hline & $\mathbf{N}^{\circ}$ de aves infestadas & $\%$ & $\%$ & Rango \\
\hline \multicolumn{5}{|l|}{ Acaros } \\
\hline Allosathes sp. & 41 & 80,4 & 11,3 & $1-69$ \\
\hline Trombicula sp. & 8 & 15,7 & 2,2 & $1-4$ \\
\hline Amblyomma sp. & 6 & 11,8 & 8,0 & $1-38$ \\
\hline \multicolumn{5}{|l|}{ Mallophagos } \\
\hline Heptapsogaster testudo & 11 & 21,6 & 4,9 & $1-24$ \\
\hline Heptapsogaster sp. & 45 & 88,2 & 5,4 & $1-16$ \\
\hline Rhynchotura teres & 47 & 92,2 & 9,7 & $1-54$ \\
\hline Menacanthus nothoproctae & 31 & 60,8 & 4,10 & $1-30$ \\
\hline
\end{tabular}


Ectoparásitos de Nothoprocta perdicaria en Chile - D. González et al.

Tabla 2. Sexo y estadios de los ectoparásitos aislados de en Nothoprocta. perdicaria (\%) en la provincia de Ñuble (Chile)

\begin{tabular}{|c|c|c|c|c|c|c|}
\hline $\begin{array}{l}\text { Parásito } \\
\text { relación }\end{array}$ & $\begin{array}{c}\text { Hembras } \\
\mathrm{n}(\%)\end{array}$ & $\begin{array}{c}\text { Machos } \\
\text { n }(\%)\end{array}$ & $\begin{array}{c}\text { Larvas } \\
\mathrm{n}(\%)\end{array}$ & Adultos & Total & \\
\hline & & & & & & $\begin{array}{l}\text { Hembra- } \\
\text { macho }\end{array}$ \\
\hline Allosathes sp. & $250(52,7)$ & $124(26,8)$ & $88(18,5)$ & 374 & 462 & $1: 0,50$ \\
\hline Heptaptogastes testudo & $26(48,1)$ & $21(38,9)$ & $7(13,0)$ & 47 & 54 & $1: 0,81$ \\
\hline Heptapsogaster sp. & $102(41,6)$ & $105(42,9)$ & $38(15,5)$ & 207 & 245 & $1: 1,03$ \\
\hline Rhynchothura teres & $150(33,0)$ & $174(38,3)$ & $130(28,6)$ & 324 & 454 & $1: 1,16$ \\
\hline Menacanthus nothoproctae & $44(34,6)$ & $32(25,2)$ & $51(40,2)$ & 76 & 127 & $1: 0,73$ \\
\hline
\end{tabular}

llamando la atención que sólo un ave adulta poseía 38 especímenes del mencionado ácaro. De este género, en Chile se ha descrito $A$. tigrinum en estado adulto en perros ${ }^{14}$, y zorros ${ }^{15}$ ). También se han descrito A. maculatum sin especificar cual era el hospedador ${ }^{16}$, el $A$. parvitarsum en camélidos en el norte de Chile y A. testudinis en el paseriforme Turdus falklandii (zorzal).

Con excepción de $R$. teres, los otros ectoparásitos diagnosticados en el presente estudio representan nuevos aportes a la fauna partasitológica nacional y en particular para la especie de perdiz chilena, $N$. perdicaria.

\section{RESUMEN}

Entre Noviembre de 1994 y abril de 1995 en la provincia de Nuble (Chile), se capturaron 51 ejemplares de perdiz chilena (Nothoprocta perdicaria), de los que se aislaron los siguientes ectoparásitos: Rhynchothura teres $(92,2 \%)$, Heptapsogaster sp. (90,2\%), Menacanthus nothoproctae $(39,2 \%)$, Heptapsogaster testudo (19,6\%), Allosathes sp. (80,4\%), Amblyomma sp. $(11,8 \%)$ y ácaros de la familia Trombiculidae (15,7\%). Con excepción de $R$. teres, los restantes ectoparásitos diagnosticados representan el primer registro en la perdiz chilena.

\section{REFERENCIAS}

1.- FJELDSA J, KRABBE N. Birds of the high Andes. Apollo Books. University of Copenagen, Dinamarca. 1990.

2.- ROTMANN J. Biología de la Perdíz chilena. Tesis Santiago de Chile. Facultad de Ciencias pecuarias y Medicina Veterinaria. Universidad de Chile 1968.

3.- DEL HOYO J A, ELliOT A, SARGATAL J. Handbook of the birds of the world. Ostrich to Ducks.
Lynx Edicions. Barcelona, España. 2000.

4.- CRUZZAT J M. Estudio del contenido de la ingluvia y estómago muscular en la tórtola (Zenaida auriculata), codorniz (Callipepla californica) y perdiz (Nothoprocta perdicaria) en periodo estival en la provincia de Ñuble, VIII Región, Chile. 2001. Memoria de título para optar al grado de Médico Veterinario en la Universidad de Concepción.

5.- OLSEN W. Aploparaksis tinamoui n. sp. cestode (Hymenolepididae) from the chilean tinamou (Nothoprocta perdicaria KITTLITZ 1830, Tinamiformes). Rev Iber Parasitol 1970; 30: 701-18.

6.- RUBILAR L, BERTOSSI E, SKEWES O. Parasitismo gastrointestinal en la perdiz común (Nothoprocta perdicaria) en la zona de Nuble, Chile. Bol Chil Parasitol 1996; 51: 35-7.

7.- CLAY T. Mallophaga from the Tinamidae. Proc Zool Soc Lond (B) 1937; 107: 133-59.

8.- PALMA P. Slide-mounting of Lice: a Detalied Description of the Canada Balsam tecnique. The New Zeland Entomologist 1978; 6: 432-6.

9.- KRANTZ G W. A Manual of Acarology, 2 Aufl. Oregon State University Book Stores, Inc. Corvalis. 1978.

10.- CARRIKER M A Jr. Studies in neotropical Mallophaga (III). Proceedings of the United States National Museum 1944; 95: 81-233.

11.- GAUD J, ATYEO W T, BERLA H F. Acariens sarcoptiformes plumicoles parasites des tinamous. Acarologia 1972; 14: 393-453.

12.- MARSHALL A G. The ecology of ectoparasitic insects. Academic Press Inc., New York Ltd. 1981.

13.- WHARTON G W, FULLER H S. A manual of the chiggers. Memoirs of the Entomological Society of Washington Nr. 1952; 4: 40-108.

14.- TAGLE I, ALVAREZ. V. Rectificación de diagnóstico: Amblyomma tigrinum Koch en lugar de Amblyomma maculatum Koch. Bol Chile Parasit 1959; 14: 55-7.

15.- TAGLE I. Ixodoidea en Chile. Bol Chile Parasit 1971; 26: 46-9.

16.- NEUMANN L G. Ixodidae. Das Tierreich, V. T. E. Schulze (Hrsg.), im Auftrage der K. Preuss. Akad. D. Wiss. zu Berlin, Lief. 1911. 26, XVI+169 pp, 76 textfigures.

Agradecimientos: Al Dr. Jacek Dabert quienes ayudo en la determinación de los Acaros. 